\title{
Verrucous mass of the leg in an octogerian woman
}

\author{
Mariem Rekik ${ }^{1}$, Khadija Sellami ${ }^{2}$, mariem Amouri ${ }^{1}$, Saadia Makni ${ }^{3}$, Naourez Gouiaa ${ }^{3}$, \\ Tahya Boudaouara ${ }^{3}$, and Hamida Turki ${ }^{1}$ \\ ${ }^{1}$ Hedi Chaker Hospital \\ ${ }^{2}$ Hopital Hedi Chaker \\ ${ }^{3}$ Hopital Habib Bourguiba
}

November 30, 2021

\begin{abstract}
Various clinical forms of cutaneous leishmaniasis can be encountered such as: ulcerated, lupoïd, sporotrichoïd and other rare forms (eczematiform, erysipeloid, psoriasiform, verrucous, and pseudotumoral). We report an atypical presentation. Dermatologists should be aware when facing a verrucous mass, especially if the patient comes from an endemic country.

Introduction:

Cutaneous leishmaniasis (CL) is endemo-epidemic in the center and south of Tunisia. We report a verrucous form mimicking squamous cell carcinoma.

Case presentation:

A 80- year old woman with no medical record is presented to our department for a 3-month history of budding tumor on her left leg. It had started as a papule which progressively enlarged and became verrucous. Physical examination shows a painless verrucous mass measuring $5 \mathrm{~cm}$ in diameter, on the external face of the left leg's lower extremity (Figure 1). There were no palpable regional nodes. The leg lesion was biopsied (Figure 2, 3). Histopathology revealed epidermal hyperkeratosis with a dense inflammatory infiltration of lymphocytes, plasmocytes and histiocytes (Figure 2) containing intracellular basophilic small and rounded structures which turned blue after Giemsa's staining; these organisms were consistent with leishmaniaamastigotes (Fig.3). The diagnosis of verrucous and pseudotumoral cutaneous leishmaniasis (CL) was established. The patient was treated by cryotherapy once a week for 4 months with good results: the lesion has almost been completely resolved at 5 months, leaving a dyschromic scar.
\end{abstract}

Discussion:

Leishmaniasis is a protozoan infection that occurs worldwide. It has diverse clinical presentations and may represent a public health problem in endemic countries [1]. Various clinical forms can be encountered such as: the ulcerated and crusted form, the lupoïd form, the sporotrichoïd form and other rare forms (eczematiform, erysipeloid, psoriasiform, verrucous, and pseudotumoral) [2]. These uncommon presentations represent 2-5\% of CL clinical presentations [3]. Small number of cases of verrucous and tumor-like forms had been reported in previous series [2]. The most important differential diagnosis is the verrucous variant of squamous cell carcinoma [4]. The diagnosis of CL may be made using several methods. The most common ones are tissue smear and skin biopsy with Giemsa staining in order to identify Leishmania amastigotes. The culture enables to identify the specimen but PCR has a higher sensitivity. The treatment of CL can be topical or systemic. Cryotherapy alone can be efficient, particularly in the case of a small number of lesions [5]. Most of the published cases of verrucous CL were treated systemically $[2,3,4]$. 
The peculiarity of our case consists in its striking resemblance to verrucous squamous cell carcinoma which refers to the verrucous and pseudotumoral presentation, and the response to cryotherapy without need for systemic treatment.

\section{Conclusion:}

We report an atypical presentation of CL. Dermatologists should be aware of this form of CL when facing a verrucous mass, especially if the patient comes from a CL endemic country.

Author contribution:

Rekik Mariem: Have made substantial contributions to conception and design, or acquisition of data, or analysis and interpretation of data; and have been involved in drafting the manuscript or revising it critically for important intellectual content; and Agreed to be accountable for all aspects of the work in ensuring that questions related to the accuracy or integrity of any part of the work are appropriately investigated and resolved.

Sellami Khadija: Have made substantial contributions to conception and design, or acquisition of data, or analysis and interpretation of data; and have been involved in drafting the manuscript or revising it critically for important intellectual content; and Agreed to be accountable for all aspects of the work in ensuring that questions related to the accuracy or integrity of any part of the work are appropriately investigated and resolved.

Amouri Meriem: Have been involved in drafting the manuscript or revising it critically for important intellectual content; and Agreed to be accountable for all aspects of the work in ensuring that questions related to the accuracy or integrity of any part of the work are appropriately investigated and resolved.

Makni Saadia: Agreed to be accountable for all aspects of the work in ensuring that questions related to the accuracy or integrity of any part of the work are appropriately investigated and resolved.

Gouiaa Naourez: Agreed to be accountable for all aspects of the work in ensuring that questions related to the accuracy or integrity of any part of the work are appropriately investigated and resolved.

Boudaouara Tahya: Agreed to be accountable for all aspects of the work in ensuring that questions related to the accuracy or integrity of any part of the work are appropriately investigated and resolved.

Turki Hamida: Have made substantial contributions to conception and design, or acquisition of data, or analysis and interpretation of data; have been involved in drafting the manuscript or revising it critically for important intellectual content; have given final approval of the version to be published and agreed to be accountable for all aspects of the work in ensuring that questions related to the accuracy or integrity of any part of the work are appropriately investigated and resolved.

\section{References:}

1. Conrad NL, Hanson SG, Hsu S.What caused this verrucous plaque?Postgrad Med. 2001 Mar;109(3):139-41.

2. Meireles CB1, Maia LC1, Soares GC1 et.al. Atypical presentations of cutaneous leishmaniasis: A systematic review.Acta Trop. 2017 Aug;172:240-254

3. Rodríguez-Zúñiga MJM1,2, Idrogo-Bustamante J1,2, Quijano-Gomero E1. Verrucous cutaneous leishmaniasis: unusual presentation. Rev Peru Med ExpSaludPublica. 2017 Apr-Jun;34(2):344-345

4. Salman A, Yucelten AD, Seckin D, et al.Cutaneous leishmaniasis mimicking verrucous carcinoma: A case with an unusual clinical course.Indian J DermatolVenereolLeprol. 2015 Jul-Aug;81(4):392-4

5. Masmoudi A1, Hariz W, Marrekchi S. et al. Old World cutaneousleishmaniasis: diagnosis and treatment.JDermatol Case Rep. 2013 Jun 30;7(2):31-41. 


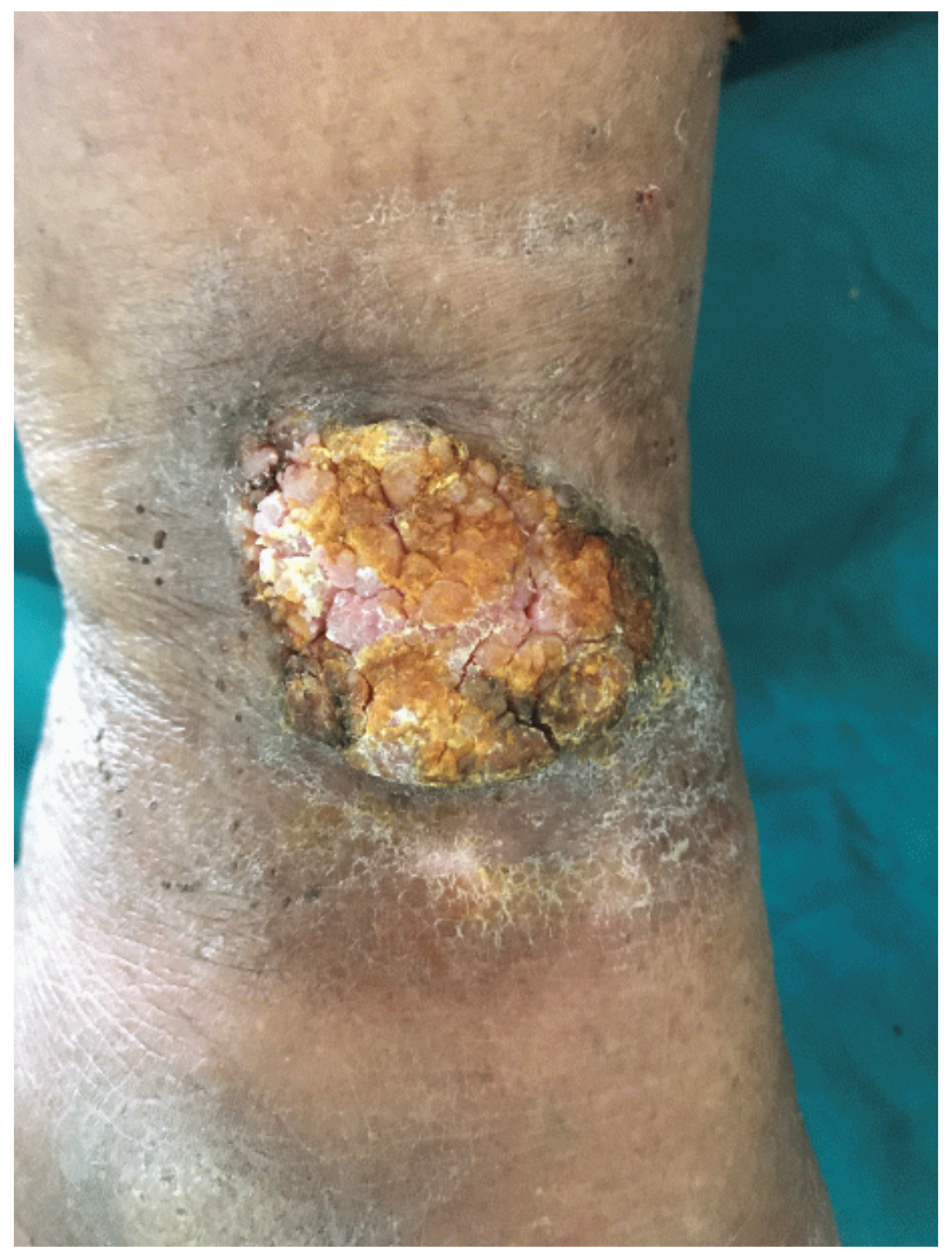



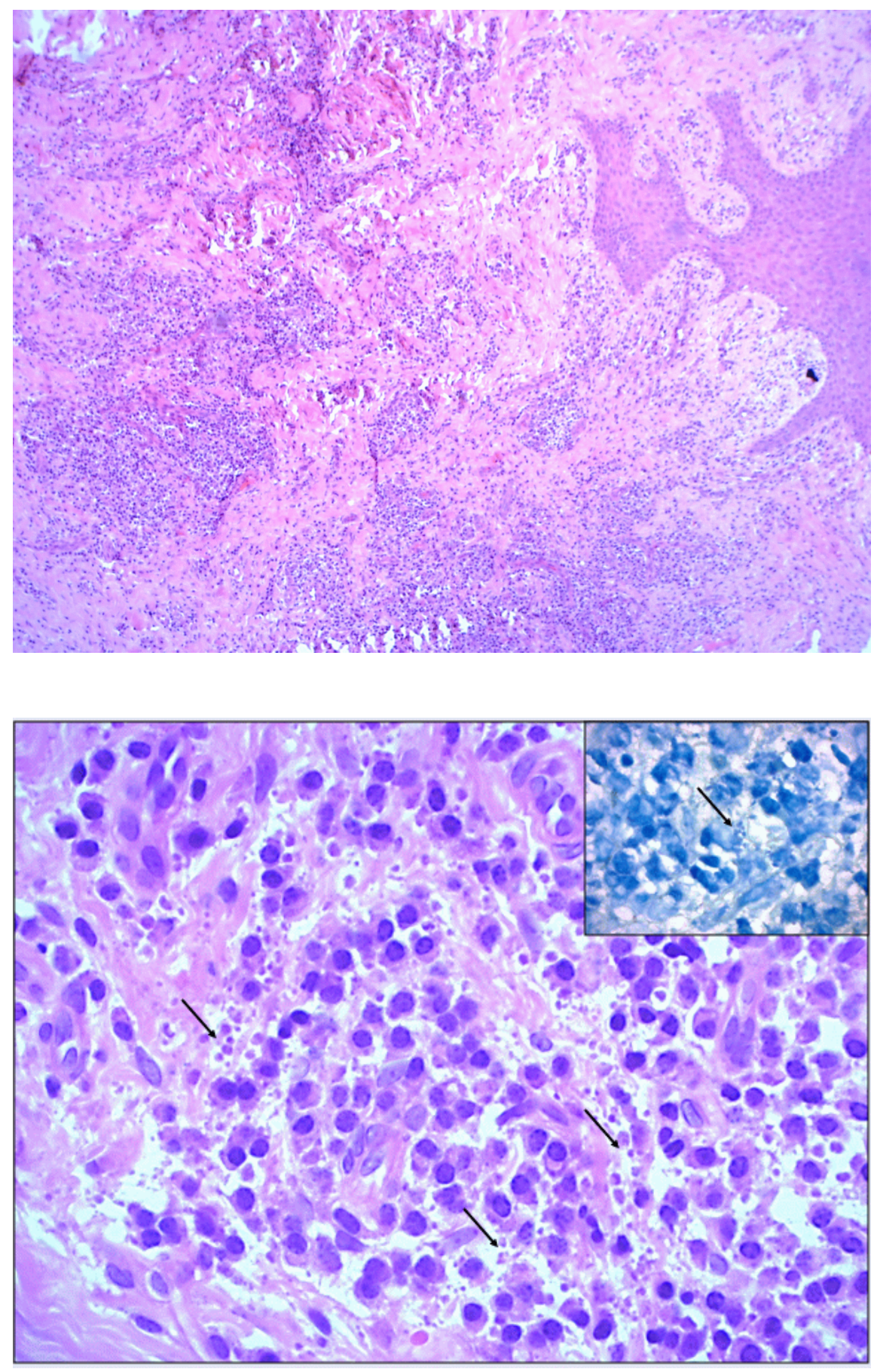